\title{
Violencia contra las mujeres en El Salvador
}

\author{
Laura Navarro Mantas \\ laurana@ugr.es \\ Universidad de Granada, España
}

\section{Resumen}

El presente artículo es una revisión teórica del problema de la violencia de género en El Salvador, y tiene el propósito de aproximarse a una construcción social del fenómeno desde la realidad del país en el que se enmarca. No en vano, la violencia contra las mujeres en El Salvador supone un grave problema de salud pública y derechos humanos. En concreto, resulta particularmente alarmante teniendo en cuenta el clima de violencia y discriminación social de la mujer que se conjugan entre sí, dando lugar a una situación de absoluta desprotección para ellas tanto fuera como dentro de sus hogares. En la actualidad, la violencia contra las mujeres supone a su vez uno de los más graves problemas políticos y sociales del país, que se une a las serias carencias que, además, sufren las mujeres en aspectos básicos como la alimentación, la educación y la higiene. Sin embargo, a pesar de la evidente magnitud del problema, en la actualidad no existen datos reales que permitan tener un conocimiento ajustado y preciso del fenómeno de cara a una mayor sensibilización y abordaje desde las políticas públicas y una adecuada asistencia a las víctimas. Finalmente, se concluye incidiendo en la importancia de llevar a cabo más investigaciones sobre este tema que permitan una mayor visibilización de la violencia contra las mujeres en El Salvador.

Palabras clave: Violencia de género, El Salvador, problema social, investigación social, políticas, violencia contra la mujer.

\section{Abstract}

This article is a theoretical review of the problem of gender violence in El Salvador, and is intended to make a social construction of the reality of the phenomenon of the country in which it is framed. Not surprisingly, violence against women in El Salvador is a serious public health and human rights problem. This is particularly alarming, given the combination of a climate of violence and social discrimination against women, resulting in a situation of an absolute lack of protection for them, both outside and inside their homes. Today, violence against women has become one of the most serious political and social problems of the country, in addition to serious deficiencies that are also suffered by women in the fulfillment of basic issues, such as food, education and hygiene. However, despite the apparent magnitude of the problem, there are currently no real data to allow for a close and precise knowledge of the phenomenon facing the increased awareness and approach from public policy and adequate assistance to victims. Finally, the article conclude stressing the importance of conducting more research on this issue to allow greater visibility of the violence against women in El Salvador.

Keywords: Gender violence, El Salvador, social problem, social research policies, violence against women. 


\section{Violencia contra las mujeres en El Salvador}

El Salvador es uno de los países con mayor tasa de homicidios de América Latina. Junto con Colombia, Guatemala y Jamaica, forma parte del grupo de países más violentos de este continente (Buvinic et al., 1999). El hecho de haber sufrido una larga guerra civil, de la que aún en la actualidad arrastra las consecuencias de la posguerra, y un presente marcado por una realidad social unida al fuerte fenómeno migratorio, las padillas y el narcotráfico, lo hace especialmente vulnerable a experimentar una violencia generalizada que se expresa a todos los niveles de la sociedad. El fácil acceso a las armas, la extrema desigualdad social y los altos niveles de pobreza de gran parte de la población, son factores que contribuyen al mantenimiento de estos altos índices de violencia.

La cultura, por otro lado, es otro de los factores responsables de la proliferación de la violencia en América Latina en general. La cultura establece unas normas y patrones de funcionamiento social que aprueban la violencia en determinados contextos. Es por ello que los valores culturales en la sociedad de El Salvador, eminentemente machista, adquieren una importancia fundamental en la proliferación y el mantenimiento de uno de los problemas más graves del país en la actualidad: la violencia contra las mujeres. Unas normas de funcionamiento social fundamentadas en los rígidos papeles de género siguen respaldando el "derecho" del marido de controlar y corregir a su pareja; y le da autoridad, por tanto, para hacer uso de la violencia contra ella (Buvinic et al., 1999; Hume, 2008). Gran parte de estas ideas tradicionales sitúan a la mujer en una posición de complementariedad y subordinación del hombre, conformando una identidad femenina a la que se le otorga la condición de "naturaleza"; un papel de género femenino fundamentado en unos pilares básicos que son la maternidad, la afectividad, el cuidado a los demás y una sexualidad heterosexual y monógama donde la virginidad aún sigue siendo un valor considerado socialmente (Garaizabal y Vázquez, 1994).

Las mujeres salvadoreñas, por tanto, han sido tradicionalmente las principales víctimas, tanto de la violencia social como de la que se produce en el ámbito privado. Desde la inseguridad ciudadana, en la que son especialmente vulnerables en unas ciudades donde los espacios públicos están hechos para los hombres y donde la mujer es relegada a lo privado y al cuidado del hogar y la familia, hasta el maltrato del que es víctima en la intimidad de su contexto más cercano. Así, la "mujer pública" tiene una connotación negativa y coloca a la mujer en una situación de riesgo en los espacios públicos urbanos, manifestándose a través de la delincuencia, el acoso laboral o la agresión sexual, entre otros (Massolo, 2005). Por otro lado, el maltrato a la mujer por su pareja es una práctica muy común en El Salvador, y está muy enraizado en la cultura y sociedad salvadoreñas, hasta el punto de que en muchos entornos está considerado como un hecho "normal" (Hume, 2004). Como consecuencia, la mujer salvadoreña se encuentra en una situación de especial vulnerabilidad en el seno de sus hogares donde la violencia es bastante aceptada (Hume, 2008). Existe, por tanto, una fuerte complicidad social con este comportamiento $y$, en general, se considera que es un asunto privado en el que nadie debe inmiscuirse, e incluso que es un derecho del marido. La sospecha sobre la mujer que es maltratada de que ella haya hecho algo para merecérselo es una creencia muy extendida, lo cual permite justificar el maltrato y alimenta el propio sentimiento de culpabilidad de la mujer que está siendo víctima de violencia (Garaizabal y Vázquez, 1994). La sociedad salvadoreña asume la supremacía del hombre y, por tanto, acepta su derecho a hacer mantener su dominación como justificación del maltrato. Se considera que, de no hacerlo, iría contra el bienestar familiar, poniendo en riesgo la misma institución del matrimonio y la familia, lo que sería sancionado socialmente (Gaborit, 2005).

Por otra parte, esta idea generalizada de que el maltrato hacia la mujer es necesario, e incluso merecido, acarrea consecuencias graves en la autoestima de la mujer en la medida en que no se siente buena madre y esposa, y le genera importantes sentimientos de culpabilidad. La religión, a su vez, tiene un gran peso en la sociedad salvadoreña, y se constituye en una ideología que suele estar basada en un fuerte sentimiento de culpa, lo que alimenta aún más este sentir en la mujer en El Salvador (Garaizabal y Vázquez, 1994). Por ende, los líderes religiosos dictan unas normas muy estrictas de comportamiento que modulan las relaciones entre mujeres y hombres. Para muchas mujeres salvadoreñas, la religión puede suponer una vía de escape y un refugio, pero, para otras muchas, es un sistema de presión 
más que mantiene los papeles de género bien diferenciados y alimenta el mencionado sentimiento de culpabilidad.

La violencia contra la mujer, en todas sus manifestaciones, ocurre en el marco de una cultura de estilo patriarcal que, al ensalzar los valores masculinos sobre los femeninos, obstaculiza la concienciación social sobre la problemática y, por tanto, la legislación al respecto y la asistencia a las víctimas. Como consecuencia, tampoco existe una estimación más o menos aproximada de la incidencia real de los casos de violencia y muerte de mujeres a manos de sus parejas. Muchos datos son ocultados, ignorados 0 disfrazados bajos otros tipos de violencia (Massolo, 2005). No obstante, a pesar de la escasez de datos reales, sí existen algunas cifras orientativas que ofrecen algunos informes de instituciones públicas, organizaciones internacionales 0 asociaciones no gubernamentales del país.

\section{Algunos datos de prevalencia de violencia de género en El Salvador}

Una dificultad añadida al esfuerzo de cuantificación de casos reales de violencia de género en El Salvador es el enfoque que se emplea desde las instituciones públicas y privadas, el cual, como señala el PNUD (2011), a menudo adolece de perspectiva de género. Así, la diversidad de terminología empleada no adecuada al problema social que estamos tratando no otorga la importancia y la especificidad de la que se hace necesario su abordaje. De esta forma, empleando términos como violencia intrafamiliar o violencia sexual se ocultan en muchos casos el maltrato físico, psicológico y/o sexual que sufre la mujer que está siendo maltratada por su pareja, que a su vez es un tipo de violencia de género cuya definición más global es la que la Organización de Naciones Unidas (ONU) (1995) dicta como "todo acto de violencia basado en la pertenencia al sexo femenino que tiene o puede tener como resultado un daño físico, sexual o psíquico para la mujer, así como las amenazas de tales actos, la coacción o la privación arbitraria de libertad, ya sea que ocurra en la vida pública o en la privada". Así, como señala la ONU, se trata de una violencia que sufren las mujeres por el mero hecho de ser mujer, es decir, por su pertenencia al género femenino, y que por definición sería diferente a la violencia intrafamiliar, que es toda violencia que se da dentro del núcleo familiar y hacia cualquier miembro de la unidad familiar.

Sin embargo, a pesar de las mencionadas dificultades para rescatar los casos de violencia de género de las estadísticas globales, el PNUD (2011) informa que en los últimos cinco años las cifras promedio de violencia intrafamiliar, cuyas víctimas han sido mujeres, superan el $90 \%$. Por otra parte, se puede afirmar que la mayoría de las víctimas de crímenes violentos en El Salvador son mujeres, y se estima que unas 1.000 han sido asesinadas entre 1999 y 2005, según un estudio de una coalición de organizaciones que combaten la violencia contra las mujeres financiada por Oxfam América (Oxfam Internacional, 2005). Este estudio informa que, en los primeros cuatro meses de 2004, se registraron 1.054 casos de violencia doméstica en la Oficina de Asuntos de la Mujer. De ellos, casi un $94 \%$ de las víctimas eran mujeres, y solo un $20 \%$ de los casos fueron llevados a los tribunales. Otros organismos públicos, como ISDEMU (Instituto Salvadoreño para el Desarrollo de la Mujer), informan que el $60 \%$ de las 7.582 denuncias registradas entre la segunda mitad de 2004 y la primera mitad de 2005, corresponden a violencia intrafamiliar, de las cuales el 93,23\% fueron interpuestas por mujeres. A su vez, en relación con las denuncias de las mujeres por violencia intrafamiliar, se registró durante el período de 2005 un incremento del $22 \%$ con respecto al año anterior (ISDEMU, 2005). Esta misma fuente gubernamental sostiene que siguen en aumento los casos, como evidencia las 3.804 denuncias por violencia intrafamiliar de 2002 frente a las 6.711 de 2009 , siendo un $97,5 \%$ de las denuncias de este último año en las que las víctimas fueron mujeres. Las estadísticas disponibles del Instituto de Medicina Legal de la Corte Suprema de Justicia de El Salvador evidencian que la mayoría de los casos de violencia física intrafamiliar contra las mujeres son cometidos por el esposo, compañero o excompañero de vida. Así lo refleja el $68,2 \%$ de los casos registrados en el año 1999, y el 70,7\% un año después. Isdemu, por su parte, identifica a la pareja masculina como maltratadora en el $85,8 \%$ de los casos registrados entre 2001 y 2003; y Ormusa (2009), el $87 \%$ de los casos registrados entre enero y septiembre de 2009. En torno a un tercio de las lesiones mostradas por las mujeres fueron manchas o señales en la piel como consecuencia de los golpes; cerca de un 10\% sufría heridas de diversa índole, y aproximadamente un 5\% mostró hematomas (Gaborit, 
2005). En un estudio publicado recientemente, se encontró que las mujeres salvadoreñas que eran maltratadas físicamente eran víctimas de una gran variedad de agresiones, incluyendo los niveles más graves. Así, un 18,4\% declaró haber sido amenazada con algún arma por parte de su pareja, y un 17,2\% había necesitado asistencia médica por las lesiones sufridas (Sierra et al., 2009). Por otro lado, parece ser que son las mujeres salvadoreñas más jóvenes el foco principal de violencia en el hogar. La Encuesta Nacional de Salud Familiar (2002-2003) identificó que el 54,9\% de las víctimas eran mujeres de edades comprendidas entre los 15 y 29 años. La Policía Nacional Civil, por su parte, informó que más de la mitad de los casos de violencia investigados fue contra mujeres menores de 30 años. En cualquier caso, son las mujeres que aún están estudiando, las que tienen un bajo nivel educativo y las analfabetas las más vulnerables a sufrir violencia. Pero son, sobre todo, las amas de casa las mujeres que con más frecuencia reciben maltrato en sus hogares, como argumenta Isdemu, con una tasa del $65,3 \%$ de mujeres atendidas sin un trabajo remunerado (Gaborit).

\section{Sensibilización social del problema de la violencia de género}

La Asociación de Mujeres por la Dignidad y la Vida de El Salvador (2006) llevó a cabo una investigación donde se analizó el tratamiento que la prensa le daba al problema de la violencia contra de la mujer en el país. Se encontró que el $87,2 \%$ de los casos de violencia de género registrados eran homicidios y casos de violencia sexual, y la prensa solo dio cobertura a los casos de extrema violencia, pasando por alto los casos de violencia física y psicológica. Con estos datos, esta asociación de mujeres denuncia que la falta de información sobre estadísticas reales respecto a la violencia contra las mujeres en El Salvador obstaculiza la visibilización de la verdadera magnitud del problema. A su vez, desde distintos sectores feministas, llaman la atención sobre el hecho de que los datos ofrecidos por los organismos públicos a menudo difieren mucho de los recopilados por asociaciones y movimientos de mujeres no gubernamentales, tanto nacionales como internacionales, que constatan una mayor gravedad del asunto.

Por otro lado, no son menos sorprendentes los resultados obtenidos en un sondeo nacional realizado por el Instituto
Universitario de Opinión Pública de la Universidad Centroamericana José Simeón Cañas (2005), que en el año 1999 revelaba datos alarmantes sobre las actitudes hacia la violencia y el desconocimiento de las medidas adoptadas por parte de la población salvadoreña. Por ejemplo, un $32 \%$ de la población femenina consideraba que es el maltrato o la violencia intrafamiliar el problema que más afecta a las mujeres del país. Sin embargo, el 84,2\% reconocía no conocer servicios del gobierno dirigidos a mujeres; y un $29,3 \%$ consideró que estos servicios eran poco efectivos. El 41,8\% reconoció no saber dónde acudir en caso de tener que hacer una denuncia sobre la violación de algún derecho como mujer. Con respecto a las actitudes hacia el marido, el $70,4 \%$ consideraba que la mujer debe estar dispuesta siempre a complacer a su marido; y el $63,6 \%$ indicaba que la mujer debe conformarse con lo que el compañero le da. Finalmente, más de la mitad de la población (el 53,7\%) manifestaba que la violencia en la casa es un asunto privado en el que nadie debe inmiscuirse.

En consonancia con la opinión pública general y las actitudes que muestra la población hacia la violencia contra las mujeres, existe una importante falta de sensibilización sobre este problema social también en el ámbito gubernamental salvadoreño, como evidencian la escasez de medidas políticas específicas para atajar el problema y el deficiente sistema de recopilación de estadísticas. Aún así, algunas de las acciones que se han llevado a cabo a escala nacional en los últimos años fueron la aprobación, en 1995, de la Ley contra la Violencia Intrafamiliar que contempla medidas cautelares y preventivas para proteger a las víctimas de violencia en el hogar, ya sean mujeres, niños o ancianos. Ese mismo año, El Salvador participó en el acuerdo firmado por la Convención Interamericana para Prevenir, Sancionar y Erradicar la Violencia contra la Mujer (Convención de Belem do Pará), que regula de forma explícita las medidas tomadas para combatir la violencia contra la mujer. Tres años después se suscribió a la Comisión para la Eliminación de todas las formas de Discriminación contra la Mujer, donde los estados miembros se comprometieron a poner en marcha los mecanismos legislativos, judiciales y administrativos para acabar con la discriminación de la mujer (Urquilla, 2005); y más recientemente estos esfuerzos culminan con la aprobación, el 25 de noviembre de 2010, de la Ley Especial Integral para una Vida Libre de Violencia para las Mujeres. 
No obstante, si estos compromisos internacionales no van acompañados de otras medidas legales y políticas en el ámbito nacional con una evidente concienciación de género corren el riesgo de perder su eficacia. Y este hecho, a menudo, tiene un claro reflejo en la insuficiente respuesta de las instituciones salvadoreñas hacía las víctimas de violencia de género, o al tratamiento que se le da a estos casos desde los medios de comunicación. Así, por ejemplo, la policía, que, frecuentemente, es la primera en intervenir en los casos de violencia, tiene una respuesta inadecuada, siendo muy extendidos el uso de estereotipos y mitos de tipo machista entre dichos profesionales. La resistencia al cambio y la falta de aplicación de los nuevos procedimientos legales convierten en ineficaces las medidas tomadas para hacer frente al problema (Sagot, 2005).

\section{Conclusiones}

A pesar de lo alarmante de las cifras expuestas a lo largo de este artículo, aún en la actualidad la violencia contra las mujeres en El Salvador no es considerada un problema grave por la población general del país (Madrigal y Tejeda, 2009). Bien sabido es que la guerra civil tuvo graves consecuencias para la población salvadoreña y que el alto grado de violencia se mantuvo durante los años de la posguerra; sin embargo, el presente no es más alentador. La violencia sigue estando a la orden del día, y la crisis mundial, el fenómeno tan extendido de la emigración y la pobreza afectan gravemente al país. Tanto es así que se ha registrado un mayor índice de asesinatos en 2005 que el de 1989. Y aunque existen por lo general más muertes de hombres que de mujeres, se ha observado un considerable aumento del número de asesinatos de mujeres entre los años 2001 y 2004 (Madrigal y Tejeda).

En síntesis, los datos a los que se ha hecho referencia en este artículo pretenden orientar sobre el grave problema que la violencia contra la mujer supone actualmente para la sociedad salvadoreña y para la salud pública de este país. Sin embargo, resultan limitados para conocer en mayor profundidad la magnitud real del fenómeno y las consecuencias que supone para las mujeres y para la población general y el desarrollo social, político y económico en El Salvador. De ahí la necesidad urgente de llevar a cabo más investigaciones en esta línea que aumenten el conocimiento sobre la medida en que esta violencia contra las mujeres ocurre y en qué circunstancias se da, de forma que permita visibilizar el problema desmontando los mitos existentes en torno al mismo y sensibilizando a la sociedad salvadoreña sobre su gravedad. Por otra parte, los datos señalados sí desprenden algunas pistas en relación con las deficiencias del sistema para hacer frente a dicha problemática y la falta de presupuestos y recursos dirigidos a asistir y recuperar la salud y el bienestar psicológico de las víctimas. Es por todo ello que se hace indispensable y oportuno intervenir en este problema social desde la base, es decir, desde la ideología, y a todos los niveles de la sociedad, involucrando en esta empresa a todos los agentes sociales y capacitando de forma específica a los profesionales que van a trabajar directamente con estos casos. En definitiva, es necesario que toda la sociedad en su conjunto asuma su responsabilidad y actúe en consecuencia, solo de esta forma se logrará atajar el grave problema de la violencia de género desde la de raíz y se caminará hacia una sociedad más justa y libre de violencia.

\section{Referencias}

Asociación de Mujeres por la Dignidad y la Vida (2006, Marzo). Violencia en contra de las mujeres a través de la prensa. San Salvador, El Salvador: Deras, D. A., Trejo, M. T. y Rosa, C.

Buvinic, M.; Morrison, A. \& Shifter, M. (1999). Violence in Latin America y Caribbean: a framework for action. Washington, DC: Inter-American Development Bank.

Garaizabal, C. \& Vázquez, N. (1994). El dolor invisible. Una experiencia de grupos de autoayuda con mujeres salvadoreñas. Madrid: Talasa.

Gavorit, M. (2005). Los senderos del poder: violencia en contra de las mujeres. Estudios Centroamericanos, 681-682, 678-698.

Hume, M. (2004). It's as if you don't know because you don't do anything about it: gender and violence in El Salvador. Environment and Urbanization, 16, 63-72.

Hume, M. (2008). The myths of violence: gender, conflict and community in El Salvador. Latin American Perspectives, 35, 59-76.

Instituto Salvadoreño para el Desarrollo de la Mujer (2005). La salud de las mujeres en El Salvador: Morbimortalidad. Recuperado el 16 de Noviembre de 2006 de: http://www.isdemu.gob.sv/Principal/ OpcionesMenu/Investigaciones/docum/Salud_Mujer.pdf 
Instituto Universitario de Opinión Pública de la Universidad Centroamericana José Simeón Cañas (2005). La situación de la Mujer en El Salvador. Recuperado el 16 de Noviembre de 2006 de: http://www.uca.edu.sv/publica/iudop/2000/boletin2/ bol200.htm

Madrigal, L. J. \& Tejeda, W. V. (2009). Facing gender-based violence in El Salvador: Contribution from the social psychology of Ignacio Martín-Baró. Feminism Psychology, 19, 368-374.

Massolo, A. (2005). Género y seguridad ciudadana: el papel y reto de los gobiernos locales. Estudios Centroamericanos, 681-682, 643-658.

Organización de Naciones Unidas (1995). Plataforma de Acción de Beijing y la violencia contra las mujeres. Recuperado el 16 de Noviembre de 2006 de: http://www.choike.org/nuevo/ informes/3826.html.

ORMUSA (2009). Violencia intrafamiliar 2006-2009. Recuperado el 7 de Octubre de 2011 de: http://observatoriodeviolencia.
ormusa.org/indicadores/2009_Indicadores_violencia_ intrafamiliar_2006_2009.pdf

Oxfam Internacional (2005). Cambiando el legado de violencia en El Salvador. Recuperado el 10 de Noviembre de 2006 de: http:// www.oxfamamerica.org/es/noticias/noticias/salvador_legado

PNUD (2011). La igualdad y la equidad de género en El Salvador. San Salvador: 2011.

Sagot, M. (2005). The critical path of women affected by family violence in Latin American. Violence Against Women, 11, 12921318.

Sierra, J. C.; Ortega, V.; Gutiérrez-Quintanilla, J. R.; Bermúdez, M. P. \& Buela-Casal, G. (2009). Violencia de pareja en mujeres salvadoreñas: prevalencia y factores sociodemográficos de riesgo. Revista Argentina de Clínica Psicológica, 18, 115-123.

Urquilla, J. (2005). Análisis del feminicidio en El Salvador: Una aproximación para el debate. San Salvador: Ormusa. 